\title{
The size, staffing, security and siting of in-patient units
}

The last 30 years have witnessed an increase in the provision of community services to the mentally ill alongside a steady reduction in the number of acute admission beds. Modern, comprehensive community mental health services still require access to in-patient care for patients during acute episodes of illness. Present developments in mental health services, in particular the greater availability of coordinated multidisciplinary input into the care of patients both in their own homes and in various outpatient settings, have radically altered the atmosphere on these wards. They are less needed for complex assessment and rehabilitation now that much of this can be provided in the community. Increasingly their function is the care and treatment of severely disturbed individuals, many of whom are detained under the Mental Health Act. Symptom control and patient safety are prominent objectives.

These changes in practice and provision have given rise to many creative and innovative developments. There is now a wide range of inpatient units across Britain reflecting almost every conceivable variation. Size ranges from small. stand alone domestic units of 8-10 inpatients up to groups of wards based on traditional mental hospital sites with over 100 patients. Siting includes old mental hospitals,
District General Hospitals, converted residential property. Increasingly, most are within their catchment area but some are still distant. Staffing and security issues (including, sometimes, patient selection) are inevitably affected by these variations.

The Royal College of Psychiatrists recognises that the in-patient provision is an integral part of a modern comprehensive mental health service, not an add-on component. It is, therefore, convening a working party chaired by Professor Tom Burns and administered by Gary Robjent to report on the siting, structure, staffing and security of in-patient services for a modern mental health service. The mental health services would welcome communications from Members of the College and other interested parties about their experiences of in-patient units. We are particularly interested in experiences, both advantages and drawbacks, of working in innovative units. We expect to make a limited number of site visits and would be grateful for information about particularly successful or unsuccessful configurations from which lessons can be learnt.

Correspondence should be addressed to Gary Robjent at the College and will be treated in full confidence: 